\title{
Caracterización del conocimiento matemático para la enseñanza de los números racionales
}

\author{
Nielka Rojas, Universidad de Granada (España) \\ Pablo Flores, Universidad de Granada (España) \\ José Carrillo, Universidad de Huelva (España)
}

Recibido el 25 de Junio de 2012; aceptado el 11 de Octubre de 2012

Caracterización del conocimiento matemático para la enseñanza de los números racionales

\section{Resumen}

Este artículo forma parte de una investigación más amplia que pretende analizar el conocimiento matemático para la enseñanza que pone en juego el profesor al enseñar los números racionales. Presentamos la relación teórica que se está utilizando para identificar componentes de conocimiento matemático que el profesor manifiesta en su acción docente, correspondencia que se fundamenta en el modelo de conocimiento matemático para la enseñanza y en el análisis didáctico. Concluimos, ejemplificando con el análisis de episodios de clase.

Palabras clave. Conocimiento profesional, conocimiento matemático para la enseñanza, análisis didáctico, práctica docente, números racionales.

\section{Caracterização do conhecimento matemático para ensinar números racionais}

\section{Resumo}

Este artigo é parte de uma investigação mais ampla que analisa o conhecimento matemático para ensinar que põe em jogo o professor para ensinar números racionais. Apresentamos a componente teórica que estamos a utilizar para identificar as componentes do conhecimento matemático que os professores manifestam durante o processo de ensino, correspondência essa que se baseia no modelo do conhecimento matemático para ensinar e na análise didática. Concluímos apresentando alguns exemplos de análise de episódios de sala de aula.

Palavras-chave. Conhecimento profissional, conhecimento matemático para ensinar, análise didática, prática de ensino, números racionais.

\section{Characterization of mathematical knowledge for teaching rational numbers}

\section{Abstract}

This article is part of a broader investigation that analyzes mathematical teaching knowledge that brings into play the teacher to teach rational numbers. We present the theoretical relationship were used to identify components of mathematical knowledge that teachers manifested in their teaching, correspondence that is based on the model of mathematical knowledge for teaching and in the training analysis. Conclude with illustrating the class analysis episodes.

Para citar: Rojas, N.; Flores, P., \& Carrillo, J. (2013). Caracterización del conocimiento matemático para la enseñanza de los números racionales. Avances de Investigación en Educación Matemática, 4 , 47-64.

CC Sociedad Española de Investigación en Educción Matemática (SEIEM). www.seiem.es 
Key words. Professional knowledge, mathematical knowledge for teaching, didactic analysis, teaching practice, rational numbers.

\begin{abstract}
Caractérisation de la connaissance mathématique pour l'enseignement des numéros rationnels

Résumé

Cet article fait partie d'une recherche plus vaste qui essaie d'analyser la connaissance mathématique pour l'enseignement que le professeur met en jeu après avoir appris les numéros rationnels. Nous présentons la relation théorique que l'on utilise pour identifier les composants de la connaissance mathématique que le professeur manifeste au cours de son enseignement, la correspondance qui est basé sur le modèle des connaissances mathématiques pour l'enseignement et dans l'analyse didactique. Nous concluons, en démontrant par des exemples d'analyse d'épisodes de classe.
\end{abstract}

Paroles clés. Connaissance professionnelle, connaissance mathématique pour l'enseignement, l'analyse didactique, pratique de l'enseignement, les numéros rationnels.

\title{
1. Introducción
}

Uno de los problemas principales, en la actualidad, en la línea de Formación de Profesores de Matemática es estudiar el conocimiento profesional del profesorado (Cardeñoso, Flores, \& Azcárate, 2001; Godino et al., 2012), donde se busca, esencialmente, analizar su naturaleza, las características que lo conforman, el grado de conocimiento matemático que tienen y han de tener los profesores para desarrollar su labor docente. Desde estas perspectivas nuestro foco de interés se centra en el conocimiento profesional más específico, el conocimiento que dispone el profesor para la enseñanza de las matemáticas. Buscamos determinar atributos particulares del conocimiento matemático para la enseñanza que manifiestan los docentes a partir de las acciones en el aula, con la intención de profundizar sobre el conocimiento para la enseñanza que es específico del profesor de matemáticas. Estudiar el conocimiento del profesor en la práctica puede ayudarnos a interpretar mejor el trabajo docente, profundizar en su naturaleza, relacionar los conocimientos con los de otros profesores, entre otros aspectos, todo ello, encaminado a diseñar procesos de formación de profesores de matemáticas.

Para comprender el conocimiento matemático implicado en la práctica en el aula, surge la necesidad de diseñar herramientas que permitan hacer operativo el proceso de la identificación de conocimiento, de modo de profundizar en su caracterización. Para ello, en este trabajo presentamos la relación teórica establecida entre el modelo de conocimiento matemático para la enseñanza (Ball, Hill, \& Bass, 2005) y el procedimiento de análisis didáctico (Gómez, 2007; Rico 1997), que nos permite identificar conocimiento a partir de la acción docente. Culminamos el escrito con la ejemplificación del análisis detallado de algunos episodios de clase donde se introduce el tema de los números racionales.

\section{Marco teórico}

\subsection{Conocimiento matemático para la enseñanza}

El concepto de conocimiento matemático para la enseñanza surge de los estudios referentes a la práctica docente, en el ámbito matemático, y a la identificación de las 
tareas habituales que realizan los profesores (Ball et al., 2005). La atención por estudiar el conocimiento matemático para la enseñanza es una preocupación constante de los estudiosos en Educación Matemática, donde el interés es profundizar en el conocimiento profesional del profesor, llevando a diversos investigadores (Ball, 2000; Ball et al., 2005; Ball, Thames, \& Phelps, 2008; Bromme, 1994; Hill, Ball \& Schilling, 2008; Ponte \& Serrazina, 2004; Shulman, 1986, 1987) a identificar dominios de conocimiento que un profesor debe tener para desarrollar su profesión docente. Una importante contribución al estudio del conocimiento profesional de los profesores aparece en los trabajos de Shulman $(1986 ; 1987)$. Estos trabajos centran su atención en el profesor, desde una perspectiva del conocimiento del contenido para la enseñanza y materia a enseñar, intentando contrarrestar lo producido en los años 80 donde el interés se enfocaba en los aspectos generales de la enseñanza más que en el conocimiento del profesor como enseñante de un contenido.

Shulman $(1986 ; 1987)$ busca resaltar la importancia del conocimiento del contenido para la enseñanza y diferenciarlo del conocimiento del contenido que tienen otros profesionales; como matizan Ball et al. (2008), la enseñanza ha de ser un trabajo profesional con su propio conocimiento base. Los trabajos de L.S. Shulman han sido reconocidos como precursores en intentar determinar los componentes del conocimiento que debe tener un profesor para la enseñanza de su disciplina, proponiendo tres dominios de conocimiento base que se requieren para la enseñanza: conocimiento del contenido a enseñar, conocimiento didáctico del contenido y conocimiento curricular. El primer tipo de conocimiento hace alusión a la "cantidad y organización del conocimiento, como tal, en la mente del profesor" (Shulman, 1987, p. 9) e incluye la comprensión de los factores principales, por ejemplo: conceptos sobre la materia, marco explicativo de la materia. Mientras que el conocimiento didáctico del contenido ${ }^{1}$ alude, más específicamente, al conocimiento de la materia para la enseñanza, concretamente a los modos de representar y enunciar el contenido para hacerlo comprensible a los demás. Este dominio incluye comprender las características del alumno y del contexto educativo, disponer con claridad metas educativas, bases filosóficas e históricas, identificar el grado de dificultad en el aprendizaje de determinados temas. Por último, el conocimiento curricular queda constituido por el conocimiento de materiales instruccionales válidos para enseñar diversos tópicos, por ejemplo, los programas y materiales diseñados para la enseñanza que sirven al profesor de herramientas para presentar el contenido de la disciplina.

Los dominios del conocimiento para la enseñanza han sido sometidos a diversas críticas y revisiones por parte de los investigadores; sin embargo, siguen siendo reconocidos como herramientas para diferenciar los componentes de conocimiento para la enseñanza que ha de tener un profesor y que pueden concernir para una enseñanza efectiva. Esto permite que surjan grandes ideas y modelos relacionados con el conocimiento profesional del profesor en el contexto de la enseñanza de las matemáticas, como referencia para algunos investigadores (Ball et al., 2005; Ball et al., 2008; Grossman, 1990; Hill et al., 2008; Krauss et al., 2008; Ponte, 1994) que han seguido profundizando en estas directrices.

Para efecto de nuestro trabajo prestamos atención a los estudios desarrollados por el grupo de investigación en Educación Matemática de la Universidad de Michigan, liderado por Deborah Ball, que estudian la naturaleza del conocimiento matemático

\footnotetext{
${ }^{1}$ Traducción de la expresión en inglés Pedagogical content knowledge. En la literatura en español es frecuente la traducción a conocimiento didáctico del contenido, como nos referimos en este trabajo.
} 
necesario para enseñar y cómo éste ayuda en el trabajo de la enseñanza, estableciendo una base práctica basada en el conocimiento matemático para la enseñanza $\left(\mathrm{MKT}^{2}\right)$, que definen como "una clase de conocimiento profesional de las matemáticas diferente del exigido en otras intensivas ocupaciones matemáticas (por ejemplo, física, contabilidad)" (Ball et al., 2005, p. 17). Estos investigadores conceptualizan la noción de conocimiento matemático para la enseñanza como "el conocimiento matemático que los profesores utilizan en el aula para producir aprendizaje y crecimiento en los alumnos" (Hill et al., 2008, p. 374). Desde esta perspectiva, podemos distinguir este conocimiento como específico y propio de los profesores, dado que implica, por ejemplo, analizar los errores de los alumnos, examinar las estrategias utilizadas para la resolución de una tarea matemática, explicar a los alumnos cuando no comprenden, saber responder a cuestiones matemáticas, evaluar las cualidades de los materiales de enseñanza, disponer de representaciones, disponer de recursos para explicar un concepto y explicitar argumentos sólidos para evidenciar que un procedimiento funciona. Por lo cual, las tareas del profesor exigen no solo conocimiento de la materia que enseña, sino también un conocimiento que es específico para desarrollar su labor de enseñanza ${ }^{3}$.

Los estudios del equipo de investigación mencionado han logrado caracterizar con detalle el conocimiento matemático para la enseñanza, basándose en los componentes del conocimiento profesional propuesto por L.S. Shulman. Ellos plantean un modelo de conocimiento matemático para la enseñanza distinguiendo dos grandes dominios de conocimiento: (a) conocimiento del contenido y (b) conocimiento didáctico del contenido, proponiendo una división de los dominios en tres subdominios específicos.

(a) El conocimiento del contenido está compuesto por tres subdominios de conocimiento. El conocimiento común del contenido (CCK) es descrito como el "conocimiento matemático y habilidades que se emplean en situaciones que no son exclusivas de la enseñanza" (Ball et al., 2008, p. 399), incluye el conocimiento que el profesor pone en juego para resolver problemas matemáticos, operar correctamente y aplicar definiciones y propiedades. El conocimiento especializado del contenido (SCK) alude al "conocimiento matemático y habilidad exclusiva para la enseñanza" (Ball et al., 2008, pp. 400-401). El profesor, para desarrollar las tareas de enseñanza, requiere un conocimiento que le permita participar en tal actividad, incluyendo, por ejemplo, representar las ideas de manera clara a los estudiantes, proporcionar explicaciones matemáticas precisas y adecuadas, examinar o comprender métodos excepcionales de resolución de problemas (Ball et al., 2005). $\quad$ El conocimiento del horizonte matemático (HCK) es definido como "el conocimiento que tiene el docente de cómo están relacionados los tópicos matemáticos incluidos en el currículo" (Ball et al., 2008, p. 403). Puede considerarse como el conocimiento sobre las relaciones entre los distintos temas matemáticos y la forma en que el aprendizaje de los temas evoluciona en los distintos niveles escolares. En términos de lo que definen Martínez, Giné, Fernández, Figueiras y Deulofeu (2011), puede entenderse como aquellas relaciones que enlazan los conocimientos previos y futuros, permitiendo, por ejemplo, estudiar otras propiedades de un concepto o procedimientos en situaciones nuevas o más complejas.

\footnotetext{
${ }^{2}$ Conservamos las siglas en inglés del modelo y de cada subdominio que lo compone.

${ }^{3}$ En la actualidad estamos desarrollando una adaptación del modelo (MKT) elaborado por Ball y colaboradores, subrayando el carácter especializado del conocimiento del profesor de matemáticas (Carrillo, Climent, Contreras, \& Muñoz-Catalán, 2013).
} 
(b) Ball et al. (2008) y Hill et al. (2008) describen el domino de conocimiento didáctico del contenido como la composición de tres subdominios de conocimiento: el conocimiento del contenido y de los estudiantes (KCS) es definido como el "conocimiento del contenido que se entrelaza con el conocimiento de cómo los estudiantes piensan, saben o aprenden un contenido particular" (Hill et al., 2008, p. 375); es decir, es el conocimiento que se utiliza en tareas de enseñanza, que implica atender a un contenido específico y aspectos particulares de los alumnos. Por ejemplo, incluye el conocimiento de los errores comunes y las dificultades más habituales que presentan los estudiantes. El conocimiento del contenido y la enseñanza (KCT) queda definido como "el conocimiento que combina el conocimiento sobre la enseñanza con el matemático" (Ball et al., 2008, p. 401); es decir, abarca saber construir a partir del razonamiento de los estudiantes y las estrategias utilizadas por ellos, procesos pertinentes para tratar y corregir sus errores y concepciones erróneas. Por último, el conocimiento del currículo (KCC), alude al conocimiento de los objetivos, contenidos, fines, orientaciones curriculares, materiales y recursos disponibles para la enseñanza, que permiten al profesor guiar su práctica y seleccionar las tareas adecuadas para el aprendizaje de sus estudiantes (Ball et al., 2008, p. 391).

\subsection{Análisis didáctico}

Rico (1997) define organizadores del currículo que permiten al profesor profundizar sobre los tópicos del currículo de matemáticas con vistas a diseñar, desarrollar y evaluar unidades didácticas, y por tanto, a organizar la práctica docente. A partir del triángulo semántico de Frege, referencia-signo-sentido, Rico (1997) establece la terna: definición-representación-fenómeno, para expresar el significado de los contenidos de las matemáticas escolares. En correspondencia con ello, funda los organizadores de significado, que son: la estructura conceptual, incluyendo la evolución histórica de cada campo o de los conceptos; la diversidad de representaciones empleadas para cada sistema conceptual, además de las modelizaciones correspondiente a los conceptos; la fenomenología de los conocimientos envueltos en los conceptos, así como las aplicaciones prácticas de cada contenido.

Una vez examinado el significado del concepto se requiere estudiar su enseñanza y aprendizaje, para lo que se propone examinar la dimensión cognitiva, mediante el estudio de las limitaciones de aprendizaje relacionadas con el contenido que pueden obstaculizar el aprendizaje de los estudiantes. Son considerados los errores y las dificultades detectados usualmente en el aprendizaje de las matemáticas de cada tópico, los problemas u obstáculos de aprendizaje asociados al contenido o concepto, igualmente la secuencia de capacidades que los alumnos pueden poner en juego para resolver las tareas (camino de aprendizaje de una tarea). La dimensión de instrucción se centra en buscar materiales y recursos didácticos que se pueden emplear para la enseñanza de cada contenido, así como las tareas de enseñanza y aprendizaje.

Los organizadores permiten establecer dimensiones de reflexión sobre un contenido matemático, que tiene que considerar el profesor cuando programa e implementa sus clases. Los organizadores se han incluido en el análisis didáctico (Gómez, 2007; Lupiáñez, 2009; Rico, 1997), que se define como "el procedimiento con el que es posible explorar, profundizar y trabajar con los diferente y múltiples significados del contenido matemático escolar, para efectos de diseñar, llevar a la práctica y evaluar actividades de enseñanza y aprendizaje." (Gómez, 2007, pp. 18-19). 
De este modo, la caracterización del análisis didáctico se fundamenta en la teoría curricular que se articula a partir de cuatro dimensiones, cultural/conceptual, cognitiva, ética o formativa y social, en distintos niveles de especificación (Rico, 1997), siendo el análisis didáctico un recurso que permite al profesor organizar la actividad de enseñanza en relación a un tema matemático específico. Así, el análisis didáctico tiene por objetivo facilitar la práctica del profesor de matemáticas, de una manera sistemática y profunda, tomando en consideración el máximo de dimensiones que influyen en su actuación, mediante cuatro tipos de análisis parciales: análisis de contenido, cognitivo, de instrucción y de actuación (Gómez, 2007).

El análisis de contenido lleva a estudiar la estructura conceptual, incluyendo su evolución histórica, así como los aspectos fenomenológicos y los sistemas de representación referente al contenido matemático, estableciéndose una estructura conceptual con vista a organizar la enseñanza del contenido escolar. El análisis cognitivo examina expectativas (fines, objetivos y capacidades), oportunidades (situaciones en las que se puede producir aprendizaje enfocado hacia las finalidades) y limitaciones de aprendizaje (dificultades y errores). El análisis de instrucción organiza las tareas de enseñanza relacionadas con un contenido matemático escolar, integrando en su análisis dos consideraciones adicionales, la resolución de problemas y los materiales y recursos disponibles. Finalmente, el análisis de actuación es un análisis a posteriori que permite al profesor confrontar los aspectos planificados con lo que sucedió cuando esa planificación se puso en práctica, además describir la comprensión de los estudiantes luego de la puesta en marcha de la enseñanza del contenido, con el propósito de brindar información que le permita mejorar su planificación y, en efecto, su práctica.

\subsection{Modelo MKT y análisis didáctico}

En nuestro trabajo empleamos el análisis didáctico como una herramienta que permite al investigador tener una recopilación de información respecto al contenido. Pero además, como un medio para articular los distintos tipos de conocimiento matemático para la enseñanza con la práctica docente. Para establecer tales relaciones consideramos tres de los análisis parciales, de contenido, cognitivo e instrucción y las distintas dimensiones que lo conforman, como se ilustra en la Figura 1.

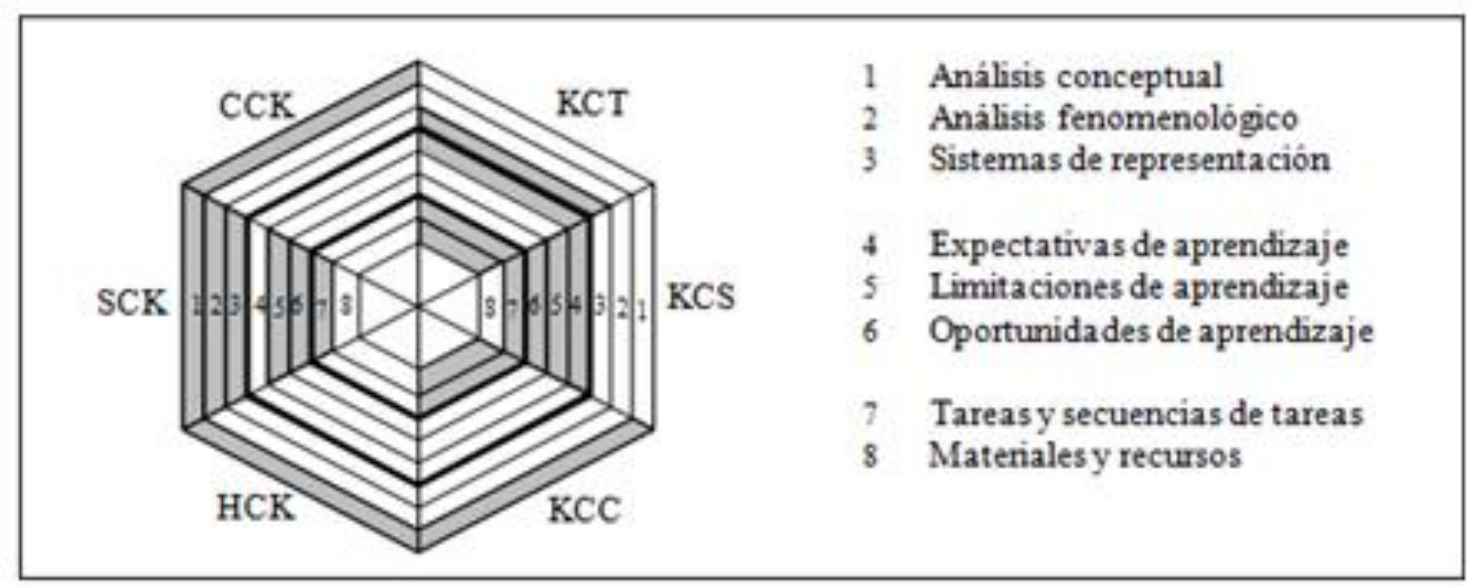

Figura 1. Relaciones entre el conocimiento matemático para la enseñanza y los componentes del análisis didáctico 
Hemos realizado el análisis de contenido del tema de los números racionales, a partir del análisis conceptual, el análisis fenomenológico y la identificación de los sistemas de representación asociado al contenido, que nos permiten organizar la enseñanza de $\mathbf{Q}$. La realización del análisis de contenido nos lleva a vincular diferentes aspectos con los subdominios CCK, SCK, HCK y KCC, como se ilustra en la Figura 1. Por ejemplo, el conocimiento común del contenido se corresponde con los conceptos y procedimientos referentes a los números racionales, como saber las operaciones en $\mathbf{Q}$, resolver ecuaciones lineales de la forma $a x+b=0, a \in \mathbf{Q}, b \in \mathbf{Q}$, ordenar los elementos de $\mathbf{Q}$, utilizar expresiones cotidianas relacionadas con las nociones de reparto equitativo o medición, emplear lenguaje de la vida cotidiana como: mitad, parte, repartir, media hora, etc.; relaciones que se establecen del análisis conceptual. El conocimiento especializado del contenido atiende a aspectos específicos para la enseñanza, como conocer los distintos significados de las fracciones, que surgen con el análisis fenomenológico del contenido. El estudio de los sistemas de representaciones lleva a conocer la diversidad de representaciones del contenido. El conocimiento del horizonte matemático deriva a conocer los distintos conceptos y procedimientos matemáticos asociados a los números racionales, como probabilidad, razones, trigonometría, semejanzas de figuras; la relación con contenidos matemáticos de niveles anteriores y posteriores al nivel que enseña el profesor; la correspondencia entre números racionales y decimales; la aplicación como porcentaje, probabilidad, razones trigonométricas, entre otras. Respecto al conocimiento del currículo lo asociamos con elementos de la estructura conceptual, específicamente el conocimiento del alcance de lo que señalan los documentos oficiales, los apartados que corresponden y las actividades recomendadas en estos.

De este modo, vinculamos los dominios de conocimiento matemático para la enseñanza con cada aspecto del análisis didáctico. Al mismo tiempo, la actuación del profesor se relaciona con algunos de los organizadores, lo que permite identificar los componentes del conocimiento (Rojas, Flores, \& Ramos, 2013). Dado a la asociación presentada, hemos elaborado categorías para identificar el conocimiento del profesor manifestado al enseñar los números racionales. Estas categorías han sido establecidas a priori, es decir, surgen antes del análisis de los datos, de modo de contar con una herramienta conceptual y operacional que facilite el análisis de la información y su posterior interpretación. Su aplicación nos está llevando a confirmar la utilidad de las mismas, asimismo a establecer nuevas categorías (emergentes) que completen las anteriores.

A continuación presentamos desarrolladas dos categorías relacionadas con el conocimiento especializado del contenido y con el conocimiento del contenido y de la enseñanza, que emplearemos en el análisis de los episodios de clase.

\section{Conocimiento especializado del contenido}

\section{Análisis conceptual}

B1. Conceptos y relaciones entre los componentes de la estructura conceptual de los números racionales (cuerpo conmutativo con las propiedades de adición y multiplicación, definido como cociente de $\operatorname{los} \mathbf{Z}, \mathbf{Q}$ denso en $\mathbf{R}$, relaciones de equivalencia y orden de $\mathbf{Q}$, existencia de neutros e inversos, etc.) 


\section{Análisis fenomenológico}

B2.1. Situaciones y contextos que ponen de manifiesto los distintos significados de las fracciones como objeto matemático, tales como:

- Las fracciones como procesos de medición y partición de una unidad.

- Situaciones que involucren las acciones de dividir, partir y repartir.

- Situaciones donde la unidad de medida no está contenida en un número exacto de veces en la cantidad que se desea medir o expresar una magnitud.

B2.2. Sentido dado a los algoritmos, según el significado empleado.

B2.3. Variedad de situaciones y contextos que aparecen en las tareas planteadas, como:

- Medidas de magnitudes (medio kilo, tres cuartos de hora).

- Relaciones concretas entre cantidades (escala de 1:1000, cartografía; tanto por ciento, comercio, por mil; falta el 10\%, descuento de un 10\%, construcción).

B2.4. Grado en que las situaciones dan sentido al contenido matemático escolar. Por ejemplo, forma de justificar el algoritmo de los productos cruzados para la división de fracciones.

\section{Sistemas de representación}

B3.1. Conocimiento explícito de la diversidad de sistemas de representación utilizados, como:

- Verbal o literal. Mitad, un medio, medio, uno de dos, media parte.

- Simbólico. División indicada $\left(\frac{1}{2}\right),(1,2)$ par ordenado, 1:2 razón, 50\% porcentaje, 0,5 decimal.

- Gráfico. Recta numerica como subconjunto de los $R$. En el plano cartesiano se puede establecer la correspondencia biúnivoca entre los puntos de cordenadas enteros, con la segunda cordenada no nula y los elementos del conjunto $\mathbf{Z} \times \mathbf{Z} /\{0\}$ y representación de las clases de equivalencia de pares de enteros.

- Figural. Una unidad dividida en partes iguales, cada parte representa una cantidad. Relación existente entre el todo (continuo o discreto) y sus partes.

- Material o concreta. Por medio de objetos, materiales y recursos (Regletas de Cuisenaire, cantidad como longitud, área o volumen, reglas de colores, Tangram, etc.).

B3.2. Establecimiento de relaciones entre los tipos de representación enunciados y realce que se concede a aquellas empleadas.

B3.3. Amplitud y precisión del lenguaje formal y algebraico utilizado según el nivel de enseñanza.

\section{Limitaciones de aprendizaje}

B4. Errores frecuentes. Saber qué conocimientos matemáticos se involucran en los errores más frecuentes de los estudiantes, en la amplitud de validez de los algoritmos y en los procedimientos no convencionales que utilizan, como por ejemplo, al sumar o restar fracciones con distintos denominadores el estudiante puede 
sumar o restar los numeradores y los denominadores entre sí. El profesor puede distinguir que ha transferido las propiedades de los $\mathbf{N}$ para realizar las operaciones con Q. Dificultades para identificar la unidad o todo según los distintos contextos del contenido. Realizar particiones no igualitarias o no exhaustivas, al aplicar una fracción a una cantidad (numérica o de magnitud, como área o longitud).

\section{Oportunidades de aprendizaje}

B5. Claridad matemática en las respuestas enunciadas a las preguntas y dudas de los estudiantes.

\section{Tareas y secuencias de tareas}

B6.1. Riqueza de las tareas matemáticas propuestas o improvisadas en el transcurso de la enseñanza, como:

- Situaciones donde la unidad de medida no está contenida un número exacto de veces en la cantidad que se desea medir o expresar una magnitud.

- Tareas sobre identificación de la unidad, de modo que los estudiantes puedan reconocer las partes en que está dividida la unidad.

B6.2. Relación de las tareas con los significados y sentidos del contenido de las fracciones y su adecuación a las situaciones y contextos más significativos.

\section{Conocimiento del contenido y de la enseñanza}

\section{Sistemas de representación}

D1.1. La adecuación entre los sistemas de representación empleados para enseñar las ideas matemáticas específicas, como las representaciones verbal, figural, numérica y material o concreta, para trabajar las cantidades como longitud y área en los niveles iniciales de la enseñanza de las fracciones, hasta llegar a representar las clases de equivalencia de pares de enteros en los niveles sucesivos.

D1.2. Ubicar la formalización rigurosa de las escrituras, los fundamentos matemáticos de las definiciones y de los algoritmos según los niveles escolares.

\section{Expectativas de aprendizaje}

D2. Objetivos de aprendizaje. Criterios para asociar los objetivos de aprendizaje planteados (implícitamente o explícitamente) con el desarrollo de la instrucción.

\section{Tareas y secuencias de tareas}

D3.1. Variedad y adecuación de los sistemas de representación que se emplean en las tareas.

D3.2. Repertorio de tareas que permiten adquirir o reforzar los conceptos matemáticos. Por ejemplo, diversidad de situaciones cotidianas que emplea para el trabajo con las fracciones como relación parte-todo.

D3.3. La evidencia de que el profesor dispone de un esquema de instrucción. Por ejemplo, comienza con una actividad de reparto, da ocasión a que actúen los estudiantes, presenta los contenidos.

D3.4. Adecuación entre las tareas presentadas y los caminos de aprendizaje que se intuyen. Como por ejemplo, en el trabajo de la relación parte-todo puede trabajarse en contextos continuos (área) e ir gradualmente incorporando actividades 
que involucren contextos discretos, o el papel que juega la representación en contextos discretos para iniciar los problemas aditivos en fracciones con distinto denominador.

\section{Materiales y recursos}

D4.1. Riqueza de materiales y recursos didácticos empleados para la enseñanza.

D4.2. Adecuación de los recursos y materiales según el nivel de enseñanza y las finalidades previstas de adquisición de conceptos y propiedades. Por ejemplo, el uso de material concreto, dibujos, para iniciar repartos equitativos.

D4.3. La relación entre acciones que permiten el empleo de recursos y situaciones, que envuelvan diversos significados y contextos. Por ejemplo, el trabajo de las fracciones como relación parte-todo puede abordarse con modelos concretos (contexto continuo, área) y luego trabajar en contextos concreto (fichas). (Transparencia de los materiales y recursos).

\section{Metodología}

Como hemos mencionado, el trabajo que presentamos es parte de una investigación más amplia donde se busca comprender, de manera profunda, el conocimiento matemático para la enseñanza que manifiestan los profesores en su tarea de enseñanza. El estudio tiene la intención de profundizar en la comprensión de una realidad singular, por lo cual hemos empleamos métodos cualitativos vinculados al paradigma interpretativo. La naturaleza de los datos es verbal, consideramos las notas de campos y para la recolección de los datos se emplean registros de baja inferencia como observaciones de audio y vídeo (Carrillo \& Muñoz-Catalán, 2011). Destacamos la observación de clase pues permite indagar detallada y sistemáticamente las características de un proceso de enseñanza.

Para ilustrar como estamos identificando conocimiento en la acción, según lo establecido, presentamos el caso de un profesor que enseña el tema de los números racionales, a estudiantes de 9 y 10 años de edad, y exponemos el análisis de algunos fragmentos de una clase.

\section{Recogida de datos}

La información sobre la enseñanza puesta en marcha y las características de la clase ejecutada por el profesor se recogen a través de la observación no participante, con un nivel de distanciamiento en las actividades de la sala de clases. La observación del aula ha sido grabada en vídeo desde la parte trasera del salón de clases con el fin de captar la totalidad del escenario y las interacciones entre el profesor y los estudiantes. Luego de la grabación y el examen de las imágenes, se transcribe la sesión; complementándose el escrito con notas (textuales) de campo, de modo que la transcripción (texto) contenga la totalidad de las interacciones entre el profesor y los estudiantes. 


\section{Análisis de datos}

El análisis se aplica al texto que registra la actividad matemática desarrollada por el docente. En el contexto del trabajo los datos obtenidos son cualitativos, el análisis se realiza a través de una descripción detallada interpretativa y se sigue el método de análisis de contenido (considerando las categorías a priori elaboradas), como técnica de investigación para la interpretación conceptual del los conocimientos que pone de relieve el profesor en su práctica (Fox, 1981; Krippendorff, 1990).

Respecto a los datos realizamos una primera división a través del criterio conversacional que consiste en separar las declaraciones o turnos de palabras u oraciones de los sujetos implicados, es decir, cuando interviene el profesor y los estudiantes (Rodríguez, Gil, \& García, 1996, p. 207). Asumimos la siguiente nomenclatura para referirnos a los sujetos involucrados: A: alumno, As: Alumnos (intervención conjunta de varios estudiantes) y P: el profesor. Posteriormente, realizamos una segunda división de los datos, esta vez considerando unidades de análisis (denominadas episodios [i], donde el índice i concierne al número de episodio) que corresponden a un fragmento que tiene un principio y un fin reconocible y una secuencia de acciones que lo constituye (Krippendorff, 1990, p. 85), teniendo en cuenta que "la unidad deber ser bastante grande como para proveer significado, al menos mediante un contexto, pero bastante pequeña como para permitir objetividad en su uso" (Hayman, 1991, p. 128). Por lo tanto, las unidades de información tienen un sentido completo, como por ejemplo la ejecución de una tarea, la validación e institucionalización de un concepto matemático, entre otros.

\section{Análisis de los datos: caracterización del conocimiento matemático para la enseñanza}

La descripción y el análisis de episodios de clase nos llevan a establecer explicaciones sobre la acción docente y, a su vez, a identificar distintos dominios de conocimiento matemático para la enseñanza que el profesor, de educación primaria, pone en juego al momento de enseñar el contenido de las fracciones.

En las Tablas 1, 2 y 3, la primera columna presenta el número de la unidad de análisis, la segunda corresponde al sujeto que interviene en la unidad, la tercera concierne al texto que da origen a los datos del estudio, la cuarta columna expone el tipo de análisis que se relaciona con el episodio correspondiente (tal relación se produce dado al contenido de la unidad), por último, la quinta columna expone el subdominio de conocimiento matemático para la enseñanza identificado, en cada unidad de análisis, según las categorías definidas.

A continuación exponemos el proceso de codificación de los datos y su posterior interpretación. Presentamos el análisis de algunos episodios correspondiente a una clase donde evidenciamos distintos tipos de conocimiento matemático para la enseñanza.

El episodio [1] se inicia con la presentación del tema a tratar y las finalidades que se esperan alcanzar (en la unidad fracción, vamos a fraccionar, leer y escribir fracciones [líneas 1, 3 y 4]). Luego, el profesor propone una tarea que comprende repartir [línea 6]. Lo anterior, conceptualmente indica que la tarea presentada permite introducir el concepto de fracción, partiendo de un problema que en $\mathbf{Z}$ se resolvería por medio de una división entera (cociente 2 y resto 1). En este caso, la tarea se presenta como un reparto que exige medir cantidades no enteras de magnitud [A1.2.]; 
se trabaja con material continuo (papel) que permite repartir el resto. Por lo anterior, observamos que el profesor manifiesta conocimiento común del contenido; además hace uso de un lenguaje riguroso al referirse al término principal, fracción [línea 1] [A1.3.] y emplea la representación verbal [línea 31]. Asimismo, cuida que la división se efectúe en partes iguales [línea 3] [A2.], manifestando dominio de la situación planteada.

Tabla 1. Episodio [1]

\begin{tabular}{|c|c|c|c|c|}
\hline In & $S u$ & Texto & *Análisis Didáctico & MKT \\
\hline 1 & $P$ & $\begin{array}{l}\text { Vamos a iniciar una nueva unidad de estudio: la } \\
\text { unidad se llama fracción. }\end{array}$ & A. Cont. Término & CCK \\
\hline 2 & As & Cómo, fracción & & \\
\hline \multirow[t]{3}{*}{3} & \multirow[t]{3}{*}{$P$} & $\begin{array}{l}\text { Qué vamos a ver, niños, hoy día vamos a ver } \\
\text { fraccionamiento de enteros en partes iguales, la }\end{array}$ & $\begin{array}{l}\text { A. Cont. Fenomenología. } \\
\text { A. Cont. }\end{array}$ & SCK \\
\hline & & escritura, la escritura de fracciones, trabajando con & Representaciones & $\mathrm{KCC}$ \\
\hline & & $\begin{array}{l}\text { papelitos lustres que les pedí ayer que trajeran.[El } \\
\text { papel lustre corresponde a un papel de color cuadrado } \\
\text { de } 10 \mathrm{cms} \text { de lado] }\end{array}$ & A. Instr. Materiales & KCT \\
\hline 4 & $P$ & $\begin{array}{l}\text { [Profesor pide a los alumnos que escriban en su } \\
\text { cuaderno][La imagen corresponde a lo que el profesor } \\
\text { escribe en la pizarra] } \\
\text { Unidad V: "Fracción" } \\
\text { Fraccionamiento, lectura y escritura de fracciones }\end{array}$ & A. Instr. Oportunidades & KCT \\
\hline 5 & $P$ & $\begin{array}{l}\text { [El profesor saca a tres alumnas adelante y explica la } \\
\text { actividad] }\end{array}$ & A. Instr. Gestión tarea & KCT \\
\hline 6 & $P$ & $\begin{array}{l}\text { Le voy a pasar a María } \underline{5 \text { papeles lustres y ella tiene }} \\
\text { que repartir entre sus dos compañeras en partes } \\
\text { iguales }\end{array}$ & $\begin{array}{l}\text { A. Cont. Fenomenología. } \\
\text { A. Instr. Tarea, gestión, } \\
\text { materiales }\end{array}$ & $\begin{array}{l}\text { SCK } \\
\text { KCC } \\
\text { KCT }\end{array}$ \\
\hline
\end{tabular}

*A.Cont.: análisis de contenido; A.Instr.: análisis de instrucción; y, A. Cogn.: análisis cognitivo

En el episodio [1] se hace uso del significado de fracción como parte todo. A partir de la acción de fraccionar [líneas 3 y 4] se introduce una tarea [línea 6] de reparto que lleva a dividir en partes iguales la unidad a repartir [B1.]. Matemáticamente hace referencia a la fracción como cociente (acción de dividir 5 objetos entre 2 personas), lo cual implica que fenomenológicamente se introduce la fracción como cociente surgiendo la noción de reparto en cantidades iguales, lo que da lugar a un fraccionamiento para terminar en una fracción relación parte-todo (medios), relacionando estos sentidos de manera explícita [línea 23, episodio [2]] (reparto, fraccionamiento, parte-todo) [B2.1.].

Por otra parte, el profesor emplea dos sistemas de representación [B3.] durante el episodio [2]: verbal o literal (dos y medio [línea 18], mitad [líneas 24, 29], un medio [línea 31], fracción [línea 28], número fraccionario [línea 32]) y concreta o material (el papel) [líneas 3 y 6 a 27]. Estas características nos permiten identificar conocimiento especializado del contenido, centrado en aspectos fenomenológicos y de representación, así como en la riqueza de las tareas. Específicamente, la tarea propuesta alude a una situación real (de repartir cantidades concretas), donde la resolución se apoya en el conocimiento informal de los alumnos como base para iniciar la enseñanza del contenido, buscando la construcción del concepto por los propios estudiantes (Llinares \& Sánchez, 1988, p. 64). 
Tabla 2. Episodio [2]

\begin{tabular}{|c|c|c|c|c|}
\hline 15 & $A$ & [Reparte cada pedazo (mitad de un papel) a cada niño] & A. Instr. Gestión tarea & KCT \\
\hline 16 & $P$ & A ver, ¿cuántos papeles enteros recibió cada niño? & A. Instr. Gestión tarea & KCT \\
\hline 17 & A & Dos, dos & & \\
\hline 18 & As & No, dos y medio [a coro los alumnos] & & \\
\hline 19 & $P$ & A ver, papeles enteros & A. Instr. Gestión tarea & KCT \\
\hline 20 & A & [una alumna] Dos enteros y dos medios & A. Instr. Respuesta & KCS \\
\hline 21 & $P$ & $\begin{array}{l}\text { Puede mostrar cuántos papeles recibió [pide a las } \\
\text { alumnas] }\end{array}$ & A. Instr. Respuesta & KCS \\
\hline 22 & $P$ & Ahí tiene cada niña, que recibió dos papeles enteros & A. Cont. Fenomenología & SCK \\
\hline 23 & $P$ & $\begin{array}{l}\text { ¿Ya, y qué se tuvo que hacer con el otro papel para que } \\
\text { cada niña recibiera lo mismo? }\end{array}$ & $\begin{array}{l}\text { A. Instr. Gestión tarea } \\
\text { A. Cont. Fenomenología }\end{array}$ & \\
\hline 24 & As & Partirlos por la mitad & & \\
\hline $\begin{array}{l}25 \\
26\end{array}$ & $\begin{array}{c}P \\
\text { As }\end{array}$ & Partirlo por la mitad, están todos de acuerdo & A. Cont. Fenomenología & SCK \\
\hline 27 & $P$ & $\begin{array}{l}\text { Cada trozo de papelito que recibió cada niña se llama } \\
\text { fracción de papel, le vamos a llamar }\end{array}$ & $\begin{array}{l}\text { A. Instr. Gestión tarea } \\
\text { A. Cont. Fenomenología }\end{array}$ & KCT \\
\hline 28 & $P$ & Pregunta: ¿qué fracción de papel recibió cada niño? & A. Cont. Oportunidad & KCT \\
\hline 29 & As & Mitad, mitad... & A. Cogn. Oportunidades & KCS \\
\hline 30 & $P$ & Bien, la mitad & $\begin{array}{l}\text { A. Cont. Concepto, } \\
\text { términos. Representación }\end{array}$ & SCK \\
\hline 31 & $P$ & $Y$ vamos a llamarle a la mitad un medio de la fracción & $\begin{array}{l}\text { A. Cont.Conceptual, } \\
\text { términos, Representación }\end{array}$ & SCK \\
\hline 32 & $P$ & Un medio es una fracción es un número fraccionario & $\begin{array}{l}\text { A. Cont. Conceptual, } \\
\text { términos, Representación }\end{array}$ & $\begin{array}{l}\text { CCK } \\
\text { SCK }\end{array}$ \\
\hline
\end{tabular}

Asimismo, se identifica conocimiento del contenido y de los estudiantes, esencialmente en las interacciones de los sujetos involucrados (profesor-alumno), que dan lugar a las oportunidades de aprendizaje [C5.2.]. Por ejemplo, el profesor refuerza qué representa cada parte del papel [líneas 20-21] y da significado numérico a la cantidad representada por cada parte [líneas 31].

La tarea de división [línea 6] da lugar a una fracción impropia (5:2) [D3.2.]. La tarea podría dificultar a los estudiantes en la identificación de la fracción como parte resultante del reparto [líneas 16-30]; sin embargo, permite repartir los trozos enteros con lo cual facilita el comienzo de la acción. Como destacan Llinares y Sánchez (1988), los alumnos realizan mejor las tareas de reparto en contextos discretos, ya que pueden emplear procedimientos directos, en el caso de la tarea [línea 6] los estudiantes comienzan repartiendo el papel. Para abordar la tarea se emplea un material manipulativo familiar (el papel) que tiene una unidad conocida por los estudiantes [D4.2.]. El material de enseñanza permite el fraccionamiento de manera sencilla a través del plegado de papel (hacer coincidir una parte con la otra) y la fracción (mitades) resultante en la tarea alude al lenguaje cotidiano. También, la tarea permite al profesor realizar variadas preguntas a los estudiantes que estimulan a dar respuestas y guían su aprendizaje [líneas 19, 21, 22, 23, 25 y 28]. Lo anterior nos lleva a establecer la presencia de conocimiento del contenido y la enseñanza, especialmente en el cuidado que el profesor pone en la enseñanza del concepto de fracción. El 
docente demuestra un lenguaje cuidadoso, que lo lleva a insistir en clarificar las condiciones en que se aplican las fracciones, el fraccionamiento y el reparto, siempre formando partes iguales [líneas 3, 6, 14], también al identificar la fracción como parte y resultado del fraccionamiento [líneas 27 y 28]. Por otra parte, explicita claramente las finalidades de su enseñanza (fraccionar, escribir y representar fracciones, [líneas 3 y 4]) [D1.2]. El profesor cuida de crear condiciones específicas de enseñanza, planteando una tarea adecuada, tanto por los datos como por las condiciones y el material empleado, además pone en práctica la tarea a través de la actuación directa de los alumnos (un repartidor y los receptores [líneas 5, 6, 17, 19 y 28]; durante el proceso estimula las respuestas y lleva a enunciarlas de manera precisa.

Observamos conocimiento del currículo ya que los contenidos tratados son propuestos por el currículo escolar de base (currículo chileno) [E1.1.]. Explícitamente, en los documentos oficiales se solicita incorporar fracciones como una forma de dar respuesta a situaciones de reparto equitativo, en las que no es posible cuantificar partes de un todo o de una unidad de medida empleando los números naturales, lo que da lugar a cuantificar trozos o partes de objetos, colecciones o unidades de medida [líneas 3 a 6]. Por otra parte, apreciamos que el profesor se guía en la enseñanza del contenido de las fracciones, por los documentos curriculares oficiales, específicamente en las orientaciones didácticas dirigidas al profesor (Ministerio de educación de Chile, 2003, pp. 142-144), que sugieren iniciar la unidad con actividades de reparto.

En el episodio [3] se continúa con la actividad de dividir en partes iguales un papel, asignando a cada parte del papel un nombre [líneas 93-96]. Se asigna una cantidad a una parte de papel, ejercitándose las representaciones numérica y verbal de la fracción [líneas 92-94-99] [A1.4.].

\begin{tabular}{|c|c|c|c|c|}
\hline 91 & $P$ & $\begin{array}{l}\text { Si. Otro papel, divídelo en cuatro partes iguales. Pégalo } \\
\text { en el cuaderno. }\end{array}$ & $\begin{array}{l}\text { A. Cogn. Oportunidad } \\
\text { A. Instr. Gestión tarea }\end{array}$ & $\begin{array}{l}\mathrm{KCS} \\
\mathrm{KCT}\end{array}$ \\
\hline 92 & $P$ & $\begin{array}{l}\text { Y recuerde hay que escribir el nombre igual que la vez } \\
\text { pasada, un número, el nombre de fracción. }\end{array}$ & $\begin{array}{l}\text { A. Cont. Concepto, } \\
\text { Representación }\end{array}$ & $\begin{array}{l}\text { SCK } \\
\text { KCT }\end{array}$ \\
\hline 93 & $A$ & Un uno, una raya y un cuatro & A. Cogn. Oportunidad & KCT \\
\hline 94 & $P$ & Muy bien. & & \\
\hline 95 & $A$ & Profesor, yo no sé partirlo en cuatro pedacitos. & & \\
\hline \multirow[t]{2}{*}{96} & $A$ & $\begin{array}{l}\text { Ya, cómo se llama cada parte } \text { [los alumnos mientras } \\
\text { pegan el papel en el cuaderno, el profesor escribe en la } \\
\text { pizarra] }\end{array}$ & $\begin{array}{l}\text { A. Instr. Gestión tarea } \\
\text { A. Cont. Fenomenología }\end{array}$ & $\begin{array}{l}\mathrm{KCT} \\
\mathrm{KCC}\end{array}$ \\
\hline & & Cada parte se llama un cuarto & & \\
\hline \multirow[t]{2}{*}{97} & $P$ & $\begin{array}{l}\text { A ver, para ir terminando por hoy. A ver, Juan, una } \\
\text { pregunta: ¿Cuántos medios tiene el entero? }\end{array}$ & $\begin{array}{l}\text { A. Cont. Fenomenología } \\
\text { A. Cont. Oportunidad }\end{array}$ & $\begin{array}{l}\mathrm{KCC} \\
\mathrm{KCT}\end{array}$ \\
\hline & & Dos & & \\
\hline 99 & $P$ & $\underline{\text { Dos medios, bien }}$ & & \\
\hline 100 & $P$ & ¿Cuántos cuartos tiene el entero? & A. Cont. Oportunidad & $\mathrm{KCT}$ \\
\hline 101 & As & Cuatro & & \\
\hline 102 & $P$ & Muy bien & & \\
\hline
\end{tabular}

De acuerdo a los documentos curriculares de base (MINEDUC, 2003) en el episodio [3] se reconstruye la unidad. El trabajo se lleva a cabo con material manipulativo donde se divide una unidad en partes iguales (medios y cuartos), a cada parte se le asigna una cantidad (fracción del papel) [líneas 89 a 102]. Se trabaja con 
fracciones unitarias; un medio, un cuarto, lo cual facilita la construcción de la unidad. Por lo anterior, identificamos conocimiento común del contenido al trabajar las fracciones, con material manipulativo, en un contexto elemental de relación partetodo [A1.2.].

Durante el episodio [3] el profesor presta atención a los cambios de representaciones, comienza de la figura que representa una unidad y al dividir obtiene partes de la unidad, y luego identifica cada parte como una fracción de papel. El profesor busca dar significado a la fracción. Fenomenológicamente se construye la unidad dando significado a la fracción como relación parte-todo (fracción del papel). Cada parte queda representada de manera simbólica [línea 94]. Lo anterior nos lleva a identificar conocimiento especializado del contenido, al presentarse dos tareas que dan sentido a la fracción como relación parte-todo [B6.1.]. De acuerdo a los sistemas de representación para las fracciones en el episodio se hace énfasis en el uso de la representación verbal, numeral y concreta [B3.], [líneas 91-94-96] estableciéndose relaciones entre ellas [B3.2.].

Trabajar las fracciones como relación parte-todo, con material concreto; conjuntamente, vincular las actividades con la construcción de la unidad a partir de los medios y los cuartos obtenidos, advierte la presencia de conocimiento del contenido y de la enseñanza, ya que existe una conjugación del contenido matemático para la enseñanza (fracción en el contexto como parte-todo) y el modo en que se busca adquirir el contenido (construcción del entero a partir de partes congruentes) [líneas 91-96] [D3.2. ]

\section{Conclusiones}

El análisis de las unidades de información nos permitió identificar distintos componentes de conocimiento matemático para la enseñanza revelado por un profesor al enseñar el contenido de las fracciones. El conocimiento especializado del contenido y el conocimiento del contenido y de la enseñanza resultaron los subdominios destacados a lo largo de los episodios presentados. Lo anterior se observa en las actuaciones del profesor cuando, por ejemplo, presenta las ideas matemáticas de manera clara e inicia la unidad de enseñanza con una tarea que le permite introducir el tema. Específicamente, identificamos conocimiento especializado del contenido cuando el profesor pone de manifiesto aspectos fenomenológicos de las fracciones, es decir al enseñar el contenido en el contexto de relación parte-todo, aludiendo implícitamente a la fracción como cociente. Además, se emplean diversos sistemas de representación (literal, simbólica y concreta), estableciéndose relaciones entre las representaciones utilizadas. Asimismo, identificamos conocimiento del contenido y de la enseñanza, ya que el profesor introduce el concepto de fracción (como relación parte-todo) a través de una situación de reparto equitativo, empleado material concreto que facilita la obtención de trozos o partes de objetos. Conjuntamente, se fortalece la lectura de diversas fracciones previamente a la introducción de la representación simbólica.

Lo anterior, manifiesta que el disponer de los análisis conceptual, cognitivo y de instrucción, permite tener un amplio referente sobre el contenido de los números racionales y de aspectos de su enseñanza y aprendizaje. De este modo, las categorías elaboradas a partir de la vinculación entre los dominios de conocimiento matemático para la enseñanza y los elementos del análisis didáctico del contenido, permiten 
comprender en detalle aspectos de un proceso de enseñanza, contemplando las distintas dimensiones que intervienen en la tarea del profesor.

Concebimos que remitirnos sólo al análisis de fragmentos de textos (unidades de análisis) restringe la identificación de conocimientos en la práctica, por lo cual consideramos necesario complementar este tipo de estudio con más análisis de episodios, entrevistas al profesor para profundizar en sus modos de proceder y así comprender si el docente es consciente del conocimiento observado.

Los procesos futuros de este estudio van encaminados a analizar un mayor número de sesiones de clase, siguiendo el proceso presentado, que nos permitan comprender el conocimiento del profesor de matemáticas al enseñar los números racionales. Asimismo, establecer categorías o descriptores precisos de conocimiento matemático para la enseñanza que permitan comprender el conocimiento manifestado en la práctica docente (Carreño, Rojas, Montes, \& Flores, 2013).

\section{Referencias}

Ball, D.L. (2000). Bridging practices. Intertwining content and pedagogy in teaching and learning to teach. Journal of Teacher Education, 51(3), 241-247.

Ball, D.L., Hill, H.C., \& Bass, H. (2005). Knowing mathematics for teaching: Who knows mathematics well enough to teach third grade, and how can we decide?. American Educator, 14-22.

Ball, D.L., Thames, M.H., \& Phelps, G. (2008). Content Knowledge for Teaching: What Makes It Special? Journal of Teacher Education, 59 (5); 389-407.

Bromme, R. (1994). Beyond subject matter: "A psychological topology of teachers' professional knowledge". En R. Biehler, R. Scholz, R. SträBer y B. Winkelman (Eds.). Didactics of Mathematics as a Scientific Discipline (pp. 73-88). Dordrecht: Kluwer Academic.

Cardeñoso, J., Flores, P., \& Azcárate, P. (2001). El desarrollo profesional de los profesores de matemáticas como campo de investigación en educación matemática. En P. Gómez \& L. Rico (Eds.), Iniciación a la investigación en didáctica de la matemática. Homenaje al profesor Mauricio Castro (pp. 233-244). Granada: Editorial Universidad de Granada.

Carreño, E., Rojas, N., Montes, M.Á., \& Flores, P. (2013 en prensa). Mathematics teacher's specialized knowledge. Reflections based on specific descriptors of knowledge. En Proceedings of the VIII CERME. Antalya, Turquía.

Carrillo, J., Climent, N., Contreras, L.C., \& Muñoz-Catalán, M.C. (2013 en prensa). Mathematics teacher specialized knowledge. En Proceedings of the VIII CERME. Antalya, Turquía.

Carrillo, J., \& Muñoz-Catalán, M.C. (2011). Análisis metodológico de las actas de la SEIEM (1997-2010) desde la perspectiva de los métodos cualitativos. Reflexión en torno a un caso. En M. Marín, B. Fernández, L.J. Blanco \& M. Palarea, (Eds.), Investigación en Educación Matemática XV (pp. 77-98). Cuidad Real: SEIEM.

Fox, D. J. (1981). El proceso de investigación en la educación. Pamplona: Eunsa.

Godino, J.D., Carrillo, J., Castro, W., Lacasta, E., Muñoz-Catalán, M.C., \& Wilhelmi, M. (2012). Métodos de investigación en las ponencias y comunicaciones presentadas en los simposios de la SEIEM. Avances de Investigación en Educación Matemática, 2, 29 -52.

Gómez, P. (2007). Desarrollo del conocimiento didáctico en un plan de formación inicial de profesores de matemáticas de secundaria. (Tesis doctoral no publicada). Universidad de Granada. España. 
Grossman, P.L. (1990). The making of a teacher: teacher knowledge and teacher education. New York: Teachers College Press.

Hayman, J.L. (1991). Investigación y Educación (3ª Ed.). Paidós Ibérica, Barcelona.

Hill, H.C., Ball, D.L., \& Schilling, S.G. (2008). Unpacking Pedagogical Content Knowledge: Conceptualizing and Measuring Teachers' Topic-Specific Knowledge of Students. Journal for Research in Mathematics Education, 39(4), 372-400.

Krauss, S., Brunner, M., Kunter, M., Baumert, J., Blum, W., Neubrand. M., \& Jordan, A. (2008). Pedagogical Content Knowledge and Content Knowledge of Secondary Mathematics Teachers. Journal of Educational Psychology, 100(3), 716-725.

Krippendorff, K. (1990). Metodología del análisis de contenido. Barcelona, España: Paidós Ibérica.

Llinares, S., \& Sánchez, M. (1988). Fracciones. Madrid: Síntesis.

Lupiáñez, J.L. (2009). Expectativas de aprendizaje y planificación curricular en un programa de formación de profesores de matemáticas de educación secundaria. (Tesis doctoral no publicada). Universidad de Granada, España.

Martínez, M., Giné, C., Fernández, S., Figueiras, L., \& Deulofeu. J. (2011). El conocimiento del horizonte matemático: más allá de conectar el presente con el pasado y el futuro. En M. Marín, G. Fernández, L.J. Blanco \& M. Palarea, (Eds.), Investigación en Educación Matemática XV (pp. 429-437). Cuidad Real: SEIEM.

Ministerio de educación de Chile (MINEDUC). (2003). Programa de Estudio. Educación Matemática. Cuarto Año de Enseñanza Básica.

Ponte, J.P. (1994). Mathematics teachers' professional knowledge. En J.P. Ponte \& J.F. Matos (Eds.), Proceedings PME XVIII (pp. 195-210). Lisboa, Portugal.

Ponte, J.P., \& Serrazina, L. (2004). Práticas profissionais dos professores de Matemática. Quadrante, 13(2), 51-74.

Rico, L. (1997). Bases teóricas del currículo de matemáticas en educación secundaria. Madrid: Síntesis.

Rodríguez, G., Gil, J., \& García, E. (1996). Metodología de la investigación cualitativa. Ediciones Aljibe.

Rojas, N., Flores, P., \& Ramos., E. (2013). El análisis didáctico como herramienta para identificar conocimiento matemático para la enseñanza en la práctica. En L. Rico, J.L. Lupiáñez \& M. Molina (Eds.), Análisis didáctico en Educación Matemática. Metodología de investigación, innovación curricular y formación de profesores. Granada, España: Universidad de Granada.

Shulman, L.S. (1986). Those Who Understand: Knowledge growth in Teaching. Educational Researcher, 15(2), 4-14.

Shulman, L.S. (1987). Knowledge and Teaching: foundations of the New Reform Harvard. Educational Review, 57(1), 1-22.

\section{Referencia de los autores}

Nielka Rojas, Universidad de Granada (España). nielka001@ gmail.com

Pablo Flores, Universidad de Granada (España).pflores@ugr.es

José Carrillo, Universidad de Huelva (España). carrillo@uhu.es 


\title{
Characterization of mathematical knowledge for teaching rational numbers
}

\author{
Nielka Rojas, Universidad de Granada (España) \\ Pablo Flores, Universidad de Granada (España) \\ José Carrillo, Universidad de Huelva (España)
}

One of the current main problems in the training of mathematics teachers as a research line is to study teacher's professional knowledge. This kind of knowledge aims to analyze the degree of mathematical knowledge teachers actually have and should have to develop their teaching, its nature and the characteristics that shape it. From this perspective, our focus of interest is the more specific professional knowledge that teachers have to teach mathematics. This article is part of a broader research which seeks to identify particular attributes of mathematical knowledge for teaching that teachers manifest in their classroom activities. We describe the process carried out to identify knowledge from teachers' actions in the classroom and exemplify the analysis of some episodes while working with rational numbers $(Q)$.

Understanding the mathematical knowledge involved in classroom practices raises the need to design tools to make more useful the process of identifying knowledge, so as to deepen its characterization. Therefore, we present the theoretical relationship established between the procedure for didactical analysis and the model of Mathematical Knowledge for Teaching (MKT). Didactical analysis allows the researcher to organize the content of rational numbers from a mathematical scope and its teaching. We perform three of the partial analysis-content, cognitive and instruction- that lead to organize the teaching of $Q$. Conducting this analysis leads to link different aspects that comprise the domains of content knowledge and pedagogical content knowledge. For example, the specialized content knowledge refers to the knowing of concepts and relationships between the components of the conceptual structure of rational numbers (like a body with commutative properties of addition and multiplication, defined as the ratio of $Z$, etc.); to know situations and contexts that reveal the different meanings of fractions as a mathematical object; and to study the diversity of representation systems used for teaching this content. These and other features are related to conceptual and phenomenological aspects, representation systems, and tasks and sequences of tasks, among others. This emerges from the relationships established between the didactical analysis and the MKT model categories that allow us to identify mathematical knowledge for teaching on $Q$ from teaching activities.

To illustrate our method of identifying knowledge in action we analyze class episodes where the topic of rational numbers is taught to students 9 or 10 years old. The description and analysis of classroom episodes take us to establish explanations for the teaching and, in turn, to identify different domains of mathematical knowledge for teaching the primary school teacher brings into play when teaching fractions. Qualitative data are collected, analysis is conducted through a detailed description, and interpretation and the method of content analysis is followed (considering a priori categories developed) as a research technique for the conceptual interpretation of the knowledge which the teacher emphasizes in practice. For example, we identify specialized content knowledge when the teacher shows phenomenological aspects of fractions and teaches the content in the context of part-whole relationship, implicitly referring to fractions as ratios. There are also various systems of representation (literal, symbolic and concrete) used, establishing relations between them.

The conceptual, cognitive and instructional analysis, provide ample models regarding the content of rational numbers and aspects of teaching and learning. Thus, the categories developed from the connection between the domains of mathematical knowledge for teaching and the elements of pedagogical content analysis allow us to understand in detail aspects of a learning process, considering the different dimensions involved in the teacher's performance. 